X. HUANG

KODAI MATH. J.

19 (1996), $17-25$

\title{
ON GEHRING-POMMERENKE'S ESTIMATE FOR QUASICIRCLES
}

\author{
XINZHONG HUANG
}

\section{Introduction}

A quasicircle can be regarded as a point of the universal Teichmüller space, which can be considered also as a set of Schwarzian derivatives by Bers' embedding theorem. In particular, we know that, if the norm of a Schwarzian derivative is less than 2 , then the derivative corresponds to a quasicircle (see, for example [11], [2], [3], [4]).

Furthermore, Gehring and Pommerenke [7] found that, if the norm of a Schwarzian derivative is less than 2 , then the complexity of the corresponding quasicircle can be estimated by the norm. The main result of this paper improves the estimate due to Gehring and Pommerenke.

We recall that, in a domain $A$, the Schwarzian derivative of $f$ is defined as

$$
S_{f}(z)=\left(\frac{f^{\prime \prime}}{f^{\prime}}\right)^{\prime}-\frac{1}{2}\left(\frac{f^{\prime \prime}}{f^{\prime}}\right)^{2},
$$

where $f(z)$ is meromorphic and locally univalent in $A$.

We see that $S_{f}$ is analytic in $A$ and satisfies

$$
S_{\varphi \circ f \circ \psi}(z)=S_{f}(\psi(z)) \phi^{\prime 2}(z)+S_{\psi}(z)
$$

for any conformal mapping $\psi$ of another domain $A^{\prime}$ onto $A$ and $\varphi \in$ Möb, where Möb denotes the group of Möbius transformations.

Let $A$ be a simply connected domain conformally equivalent to a disk. The norm of Schwarzian derivative of $f$ in $A$ is defined as

$$
\left\|S_{f}\right\|_{A}=\sup _{z \in A}\left|S_{f}(z)\right| \lambda^{-2}(z),
$$

where $\lambda$ is the Poincare density on $A$.

Next, by the transformation rule (1.2) and conformal invariance of the hyperbolic metric, we have

$$
\left\|S_{f}\right\|_{A}=\left\|S_{f \circ \psi}-S_{\psi}\right\|_{A^{\prime}} .
$$

1980 Mathematics subject classifications: Primary 30C60; Secondary $30 \mathrm{C} 75$.

Received June 2, 1994; received January 17, 1995. 
In particular, setting $g=\psi^{-1}$, we have the formula

$$
\left\|S_{f}-S_{g}\right\|_{A}=\left\|S_{f \circ g^{-1}}\right\|_{A^{\prime}} .
$$

If we choose $f$ here to be the identity mapping, we get the invariance

$$
\left\|S_{g}\right\|_{A}=\left\|S_{g^{-1}}\right\|_{A^{\prime}},
$$

between a conformal mapping and its inverse.

Now, we introduce a domain constant

$$
\delta(A)=\left\|S_{f}\right\|_{A},
$$

where $f(z)$ is a conformal mapping of $A$ onto a disk. We call $\delta(A)$ the distance of the domain $A$ from a disk. In view of (1.2), the distance $\delta(A)$ is well defined. We have $\delta(A)=0$ if and only if $A$ is a disk. The constant $\delta(A)$ measures how far a simply connected domain $A$ away from a disk.

Kraus [8] showed that, if $f(z)$ is a conformal mapping of a disk $B$, then

$$
\left\|S_{f}\right\|_{B} \leqq 6 .
$$

The bound is sharp. And from this, we have

$$
\delta(A) \leqq 6 .
$$

On the other hand, in 1949 Nehari [11] showed that, if $f(z)$ is meromorphic and locally univalent in a disk $B$ and

$$
\left\|S_{f}\right\|_{B} \leqq 2, \quad z \in B
$$

then $f(z)$ is univalent in $B$. The bound 2 is sharp.

A Jordan curve can be defined as the image of a circle under a homeomorphism of the plane. If the homeomorphism is conformal, then the image is a circle. Between the topological and proper circles, quasicircles form a very important class of curves. The Jordan curve $\Gamma$ is called a quasicircle with constant $M$ if

$$
\min \left\{\operatorname{diam} \Gamma_{1}, \operatorname{diam} \Gamma_{2}\right\} \leqq M\left|w_{1}-w_{2}\right|, \quad \text { for } w_{1}, w_{2} \in \Gamma
$$

where $\Gamma_{1}$ and $\Gamma_{2}$ are the components of $\Gamma \backslash\left\{w_{1}, w_{2}\right\}$. A domain bounded by a quasicircle with constant $M$ will be called a quasidisk with constant $M$. (This important concept was introduced by Ahlfors and appears in many different contexts (see e.g. [6]).) If $f(z)$ is univalent in $D$, then $A=f(D)$ is a quasidisk if and only if $f$ has a quasiconformal extension to $\hat{C}$ as Ahlfors [1] has shown.

To estimate $\delta(A)$ for quasidisks A, Lehto [9, p. 73] showed that, if $f(z)$ is a $K$-quasiconformal mapping of the unit disk $D$ and $A=f(D)$, then

$$
\delta(A) \leqq 6 \frac{K^{2}-1}{K^{2}+1} .
$$


From (1.11) we see that, the distance $\delta(A) \rightarrow 0$ as $K \rightarrow 1$, which means that $A$ is close to a disk. So it might be expected that domains close to a disk are quasidisks. This is indeed the case. Ahlfors and Weill [2] proved that, if a meromorphic function $f$ in a disk $B$ satisfies the condition

$$
\left\|S_{f}\right\|_{B}<2,
$$

then $f$ is univalent and can be extended to a quasiconformal mapping of the plane. The constant 2 is best possible. Moreover, if $B$ is the unit disk $D$, there is an extension with complex dilatation

$$
\mu(1 / \bar{z})=-\frac{1}{2}(z / \bar{z})^{2}\left(1-|z|^{2}\right)^{2} S_{f}(z) \quad(z \in D) .
$$

Now, in order to measure how far $f(D)$ away from the unit disk by the value of $\delta(f(D))$, Gehring and Pommerenke [7, Theorem 3] showed in 1984 that

THEOREM A. If $f(z)$ is meromorphic and locally univalent in $D$ and if

$$
\delta(f(D))=\left\|S_{f}\right\|_{D} \leqq b<2,
$$

then $f(D)$ is a quasidisk with constant

$$
M \leqq 8\left(1-\frac{b}{2}\right)^{-1 / 2}
$$

The following example ([7, p. 229]) shows that the order of the bound for $M$ is best possible as $b \rightarrow 2$.

Example. For $b<2$, let $a=(1-b / 2)^{1 / 2}$, then the function

$$
f(z)=\frac{[(1+z) /(1-z)]^{a}-1}{[(1+z) /(1-z)]^{a}+1} \quad(z \in D)
$$

satisfies (1.12) while (1.10) holds for $\Gamma=\partial f(D)$ only for

$$
M \geqq\left(2 \sin \frac{a \pi}{4}\right)^{-1} \geqq \frac{2}{\pi}\left(1-\frac{b}{2}\right)^{-1 / 2} \text {. }
$$

Thus the order of the bound for $M$ in (1.13) is best possible as $b \rightarrow 2$.

Theorem A shows a relationship between $\delta(f(D))$ and $M$. Thus it is an interesting problem to find the sharpest value of $M$ depending only on $\delta(f(D))$. In this note we improve the above Theorem $\mathrm{A}$ as follows

THEOREM 1. If $f(z)$ is meromorphic and locally univalent in $D$ and if

$$
\delta(f(D))=\left\|S_{f}\right\|_{D} \leqq b<2,
$$

then $f(D)$ is a quasidisk with constant 


$$
M \leqq 2\left[2+\left(2+t_{0}\right) \sqrt{\frac{1+\frac{b}{6}}{2\left(1+t_{0}\right)}}\right]\left(1-\frac{b}{2}\right)^{-1 / 2}<2\left(2+\sqrt{3+\frac{b}{6}}\right)\left(1-\frac{b}{2}\right)^{-1 / 2},
$$

where $t_{0}$ is the unique solution of the equation

$$
2(1+t) \exp (-t)=1+\frac{b}{6} \text {. }
$$

\section{Preliminaries}

In this section, we derive some coefficient estimates for the functions satisfying (1.14). Such problems are very important even in the research of conformal mappings.

Let $S$ be the class of functions $f(z)=z+a_{2} z^{2}+a_{3} z^{3}+\cdots$, which are analytic and univalent in $D$, and define $d_{f}(z)=\operatorname{dist}(f(z), \partial f(D))$ for $z \in D$. Let $\Sigma_{0}$ be the class of functions $F(\zeta)=\zeta+b_{1} \zeta^{-1}+\cdots$, which are analytic and univalent in $\Delta=\widehat{\boldsymbol{C}}-\bar{D}$. We need the following

LEMma 1 (Fekete-Szegö [5]). Let $f \in S$. Then

$$
\left|a_{3}-\lambda a_{2}^{2}\right| \leqq 1+2 \exp \left(-\frac{2 \lambda}{1-\lambda}\right) \text { for } \lambda \in[0,1) .
$$

This bound is sharp for each $\lambda$.

LEMMA 2 (Löwner [10]). Suppose $F \in \Sigma_{0}$, then

$$
|F(\zeta)| \leqq|\zeta|+\frac{1}{|\zeta|}
$$

for $|\zeta|>1$.

The following Lemma 3 is very useful for the proof of Theorem 1, and it is also an interesting problem concerning with coefficient region of univalent functions.

LEMma 3. Suppose that $f(z)$ satisfies the assumptions of Theorem 1 , and for $z_{0} \in D$ let

$$
F(z)=\frac{f\left(\frac{z+z_{0}}{1+\bar{z}_{0} z}\right)-f\left(z_{0}\right)}{\left(1-\left|z_{0}\right|^{2} f^{\prime}\left(z_{0}\right)\right.}=z+A_{2} z^{2}+A_{3} z^{3}+\cdots .
$$

Then $F \in S$ and

$$
\left|A_{2}^{2}-A_{3}\right| \leqq \frac{1}{6} \delta(f(D)) \leqq \frac{b}{6}<\frac{1}{3}
$$

Furthermore, 


$$
\left|A_{2}\right| \leqq\left(2+t_{0}\right) \sqrt{\frac{1+\frac{1}{6} \delta(f(D))}{2\left(1+t_{0}\right)}}<\sqrt{3+\frac{b}{6}},
$$

and

$$
d_{F}(0) \geqq \frac{1}{2+\left(2+t_{0}\right) \sqrt{\frac{1+\frac{1}{6} \delta(f(D))}{2\left(1+t_{0}\right)}}},
$$

where $t_{0}$ is the unique solution of the following equation

$$
2(1+t) \exp (-t)=1+\frac{1}{6} \delta(f(D))
$$

Proof of Lemma 3. It is easy to see that

$$
\begin{aligned}
F(z)= & z+\frac{1}{2}\left[\left(1-\left|z_{0}\right|^{2}\right) \frac{f^{\prime \prime}\left(z_{0}\right)}{f^{\prime}\left(z_{0}\right)}-2 \bar{z}_{0}\right] z^{2} \\
& +\frac{1}{6 f^{\prime}\left(z_{0}\right)}\left[f^{\prime \prime \prime}\left(z_{0}\right)\left(1-\left|z_{0}\right|^{2}\right)^{2}-6 \bar{z}_{0} f^{\prime \prime}\left(z_{0}\right)\left(1-\left|z_{0}\right|^{2}\right)+6 \bar{z}_{0}^{2} f^{\prime}\left(z_{0}\right)\right] z^{3}+\cdots
\end{aligned}
$$

Since $\left\|S_{f}\right\|_{D}<2, f$ is univalent in $D$ (Nehari [11]), and so is $F$. By calculation we have

$$
A_{2}^{2}-A_{3}=-\frac{1}{6}\left(1-\left|z_{0}\right|^{2}\right)^{2} S_{f}\left(z_{0}\right)
$$

According to (1.14), it turns out that

$$
\left|A_{2}^{2}-A_{3}\right| \leqq \frac{1}{6} \delta(f(D)) \leqq \frac{b}{6}<\frac{1}{3} .
$$

Next (2.1) implies that

$$
\left|A_{3}\right| \leqq 1+\lambda\left|A_{2}\right|^{2}+2 \exp \left(-\frac{2 \lambda}{1-\lambda}\right) \text { for } \lambda \in[0,1) .
$$

From (2.3) and (2.6) we have

$$
\begin{aligned}
\left|A_{2}\right|^{2} & \leqq\left|A_{2}^{2}-A_{3}\right|+\left|A_{3}\right| \\
& \leqq \frac{1}{6} \delta(f(D))+1+\lambda\left|A_{2}\right|^{2}+2 \exp \left(-\frac{2 \lambda}{1-\lambda}\right) .
\end{aligned}
$$

Thus

$$
\left|A_{2}\right|^{2} \leqq \frac{1}{1-\lambda}\left[1+\frac{1}{6} \delta(f(D))+2 \exp \left(-\frac{2 \lambda}{1-\lambda}\right)\right] \text { for } \lambda \in[0,1)
$$

Set $\lambda=t /(2+t)$ for $t \in[0, \infty)$ and let 


$$
\begin{aligned}
G(t) & =\frac{1}{1-\lambda}\left[1+\frac{1}{6} \delta(f(D))+2 \exp \left(-\frac{2 \lambda}{1-\lambda}\right)\right] \\
& =\frac{2+t}{2}\left[1+\frac{1}{6} \delta(f(D))+2 \exp (-t)\right] .
\end{aligned}
$$

Then we have

$$
\min _{0 \leqq t<\infty} G(t)=\frac{\left(2+t_{0}\right)^{2}}{1+t_{0}} \frac{1+\frac{1}{6} \delta(f(D))}{2}<G(0)=3+\frac{\delta(f(D))}{6} \leqq 3+\frac{b}{6},
$$

where $t_{0}$ is the unique solution of the equation

$$
2 \exp (-t)(1+t)=1+\frac{1}{6} \delta(f(D)) .
$$

Hence

$$
\left|A_{2}\right| \leqq\left(2+t_{0}\right) \sqrt{\frac{1+\frac{1}{6} \delta(f(D))}{2\left(1+t_{0}\right)}}<\sqrt{3+\frac{b}{6}} .
$$

Finally, let $\zeta=1 / z$. Since $1 / F(1 / \zeta)+A_{2} \in \Sigma_{0}$, by Lemma 2 , we have

$$
\left|\frac{1}{F(1 / \zeta)}+A_{2}\right| \leqq|\zeta|+\frac{1}{|\zeta|}
$$

for $|\zeta|>1$. Thus

$$
|F(z)| \geqq \frac{r}{1+\left|A_{2}\right| r+r^{2}}, \quad \text { for } 0 \leqq|z|=r<1 .
$$

From (2.4) and (2.8), we obtain that

$$
\begin{aligned}
d_{F}(0) & =\operatorname{dist}(0, \partial F(D)) \geqq \frac{1}{2+\left|A_{2}\right|} \\
& \geqq \frac{1}{2+\left(2+t_{0}\right) \sqrt{\frac{1+\frac{1}{6} \delta(f(D))}{2\left(1+t_{0}\right)}}}>\frac{1}{2+\sqrt{3+\frac{b}{6}}} .
\end{aligned}
$$

A domain $G \subset C$ has a $c$-accessible boundary if each $z_{1}, z_{2} \in \partial G$ can be joined by an open $\operatorname{arc} \Gamma \subset G$ such that

$$
\min _{j=1,2}\left|z-z_{j}\right| \leqq c \operatorname{dist}(z, \partial G), \quad \text { for } z \in \Gamma
$$

The following characterization of quasidisks proved by Gehring and Pommerenke [7, Lemma 1] is very useful.

LEMma 4. Let $G$ be a Jardan domain in $\boldsymbol{C}$. Suppose that there is a constant $c$ such that, for all $\varphi \in$ Möb with $\varphi(G) \subset C$, the domains $\varphi(G)$ have c-accessible 
boundaries. Then $\partial G$ is a quasicircle with constant $M \leqq 2 c$.

\section{The proof of Theorem 1}

We show that $f(D)$ has a $c$-accessible boundary. To verify (2.9), assume that $z_{1}=f(-1)$ and $z_{2}=f(1)$, for $\left\|S_{f \circ \phi}\right\|_{D}=\left\|S_{f}\right\|_{D}$ for all $\phi \in$ Möb with $\phi(D)=D$ by (1.4).

Let

$$
h(t)=\frac{e^{t}-1}{e^{t}+1} \quad(t \in T=\{t:-\pi / 2<\operatorname{Im} t<\pi / 2\}) .
$$

It maps the strip $T$ conformally onto $D$ and

$$
g(t)=f \circ h(t)
$$

is analytic and univalent in $T$. A computation shows that

$$
\left|g^{\prime}(t)\right|=\frac{1}{2}\left(1-r^{2}\right)\left|f^{\prime}(h(t))\right| \text { for } t \in R, r=h(t) .
$$

Since $S_{h}(t)=-1 / 2$, it follows that

$$
\operatorname{Re} S_{g}(t)=-\frac{1}{2}+\frac{1}{4}\left(1-r^{2}\right)^{2} \operatorname{Re} S_{f}(r) \leqq-\frac{1}{2}+\frac{1}{4}\left\|S_{f}\right\|_{D} \leqq \frac{b-2}{4} .
$$

We define

and see that

$$
v(t)=\left|g^{\prime}(t)\right|^{-1 / 2}, \quad \text { for } t \in R
$$

$$
\frac{v^{\prime}}{v}=-\frac{1}{2} \operatorname{Re} \frac{g^{\prime \prime}}{g^{\prime}}, \quad \frac{v^{\prime \prime}}{v}-\left(\frac{v^{\prime}}{v}\right)^{2}=-\frac{1}{2} \operatorname{Re}\left[\frac{d}{d t} \frac{g^{\prime \prime}}{g^{\prime}}\right]
$$

and therefore, from (3.2),

where

$$
v^{\prime \prime}(t)=p(t) v(t), \quad t \in R,
$$

$$
p(t)=-\frac{1}{2} \operatorname{Re} S_{g}(t)+\left(\frac{1}{2} \operatorname{Im} \frac{g^{\prime \prime}}{g^{\prime}}\right)^{2} \geqq \frac{2-b}{8} .
$$

We set $a=((2-b) / 8)^{1 / 2}$. And for any given $t_{*}$ we may assume that $v^{\prime}\left(t_{*}\right) \geqq 0$, otherwise we replace $g(t)$ by $g\left(2 t_{*}-t\right)$. We compare the differential inequality with the initial value problem

$$
u^{\prime \prime}(t)=a^{2} u(t)\left(t \geqq t_{*}\right), \quad u\left(t_{*}\right)=v\left(t_{*}\right), \quad u^{\prime}\left(t_{*}\right)=0 .
$$

Which is solved by

$$
u(t)=v\left(t_{*}\right) \frac{e^{a\left(t-t_{*}\right)}+e^{-a\left(t-t_{*}\right)}}{2} .
$$


Because

$$
\begin{aligned}
\frac{d}{d t} \frac{v(t)}{u(t)} & =\frac{v^{\prime}(t) u(t)-v(t) u^{\prime}(t)}{u^{2}(t)} \\
& =u^{-2}(t)\left[\int_{t_{*}}^{t}\left(v^{\prime \prime} u-v u^{\prime \prime}\right) d s+v^{\prime}\left(t_{*}\right) v\left(t_{*}\right)\right] \geqq 0, \text { for } t>t_{*} .
\end{aligned}
$$

We deduce that $v(t) \geqq u(t)$ for $t \geqq t_{*}$. Thus, by (3.5),

$$
\begin{aligned}
\int_{t_{*}}^{\infty}\left|g^{\prime}(t)\right| d t & =\int_{t_{*}}^{\infty} v^{-2}(t) d t \leqq \int_{t_{*}}^{\infty} u^{-2}(t) d t \\
& =v^{-2}\left(t_{*}\right) \int_{t_{*}}^{\infty} \frac{4}{\left(e^{a\left(t-t_{*}\right)}+e^{-a\left(t-t_{*}\right)}\right)^{2}} d t \\
& =\left|g^{\prime}\left(t_{*}\right)\right| \int_{0}^{\infty} \frac{4}{\left(e^{a u}+e^{-a u}\right)^{2}} d u \\
& =\frac{1}{a}\left|g^{\prime}\left(t_{*}\right)\right| .
\end{aligned}
$$

Suppose $z_{*} \in(-1,1)$. Let $t_{*}$ satisfy $h\left(t_{*}\right)=z_{*}$ and obtain, from (3.1) and (3.6), that

$$
\min _{j=1,2}\left|z_{j}-f\left(z_{*}\right)\right| \leqq \int_{t_{*}}^{\infty}\left|g^{\prime}(t)\right| d t \leqq \frac{1}{a}\left|g^{\prime}\left(t_{*}\right)\right|=\frac{1}{2 a}\left(1-z_{*}^{2}\right)\left|f^{\prime}\left(z_{*}\right)\right| .
$$

Let $F(\zeta)$ be the function defined by $(2.2)$ with $z_{0}=z_{*}$. Then we see that

$$
\operatorname{dist}\left(f\left(z_{*}\right), \partial f(D)\right)=d_{F}(0)\left(1-z_{*}^{2}\right)\left|f^{\prime}\left(z_{*}\right)\right|
$$

This together with (2.5) shows

$$
\begin{aligned}
\frac{1}{2 a}\left(1-z_{*}^{2}\right)\left|f^{\prime}\left(z_{*}\right)\right|= & \frac{1}{2 a d_{F}(0)} \operatorname{dist}\left(f\left(z_{*}\right), \partial f(D)\right) \\
& \leqq \frac{2+\left(2+t_{0}\right) \sqrt{\frac{1+\frac{b}{6}}{2\left(1+t_{0}\right)}}}{2 a} \operatorname{dist}\left(f\left(z_{*}\right), \partial f(D)\right) .
\end{aligned}
$$

By (3.7) and (3.8) we see that (2.9) holds with

$$
c=\left[2+\left(2+t_{0}\right) \sqrt{\frac{1+\frac{b}{6}}{2\left(1+t_{0}\right)}}\right]\left(1-\frac{b}{2}\right)^{-1 / 2} \text {. }
$$

Since $S_{\phi \circ f}=S_{f}$ for all $\phi \in$ Möb, the assumption of Lemma 4 is satisfied with $G=f(D)$ and $c$ in (3.9).

The statement now follows from Lemma 4 and (2.4). 


\title{
REFERENCES
}

[1] L.V. Ahlfors, Quasiconformal reflections, Acta Math., 109 (1963), 291-301.

[2] L.V. Ahlfors AND G. Weill, A uniqueness theorem for Beltrami equations, Proc. Amer. Math. Soc., 13 (1962), 975-978.

[3] L. Bers, Simultaneous uniformization, Bull. Amer. Math. Soc., 66 (1960), 94-97.

[4] L. BERs, Correction to "Spaces of Riemann surfaces as bounded domains", Bull. Amer. Math. Soc., 67 (1961), 465-466.

[5] M. FeKete AND G. Szegö, Eine Bemerkung über ungerade schlichte Funktionen, J. London Math. Soc., 8 (1933), 85-89.

[6] F.W. GeHring, Uniform domains and the ubiquitous quasidisk, Jahresber. Deutsch. Math.-Verein, 89 (1987), 88-103.

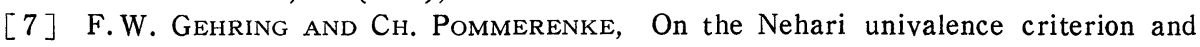
quasicircles, Comment. Math. Helv., 59 (1984), 226-242.

[8] W. KRAUS, Über den Zusammenhang einiger Charakteristiken eines einfach zusammenhängenden Bereiches mit der Kreisabbildung, Mitt. Math. Sem. Giessen, 21 (1932), 1-28.

[9] O. Lehto, Univalent Functions and Teichmüller Spaces, Springer-Verlag, New York, 1987.

[10] K. LöwNER, Über Extremumsätze bei der konformen Abbildung des Äußeren des Einheitskreises, Math. Z., 3 (1919), 65-77.

[11] Z. Nehari, The Schwarzian derivative and schlicht functions, Bull. Amer. Math. Soc., 55 (1949), 545-551.

\author{
Department of Mathematics \\ FACULTY OF SCIENCE \\ KYOTO UNIVERSITY \\ Куото 606 \\ JAPAN \\ Present Address: \\ Department of Applied Mathematics \\ HuAqIaO UNIVERSITY \\ QUANZHOU, FUJIAN, 362011 \\ P.R.C.
}

